\title{
Upper-limb robot-assisted therapy in rehabilitation of acute stroke patients: Focused review and results of new randomized controlled trial
}

\author{
Stefano Masiero, MD; ${ }^{1}$ Mario Armani, $\mathbf{M D} ;^{2}$ Giulio Rosati, $\mathbf{P h D}^{3^{*}}$ \\ ${ }^{1}$ Department of Rehabilitation Medicine, University of Padua, Padua, Italy; ${ }^{2}$ Neuromotor Rehabilitation Unit, Eremo Hos- \\ pital, Arco (Trento), Italy; ${ }^{3}$ Department of Innovation in Mechanics and Management, University of Padua, Padua, Italy
}

\begin{abstract}
The successful motor rehabilitation of stroke patients requires early intensive and task-specific therapy. A recent Cochrane Review, although based on a limited number of randomized controlled trials (RCTs), showed that early robotic training of the upper limb (i.e., during acute or subacute phase) can enhance motor learning and improve functional abilities more than chronic-phase training. In this article, a new subacute-phase RCT with the Neuro-RehabilitationroBot (NeReBot) is presented. While in our first study we used the NeReBot in addition to conventional therapy, in this new trial we used the same device in substitution of standard proximal upper-limb rehabilitation. With this protocol, robot patients achieved similar reductions in motor impairment and enhancements in paretic upper-limb function to those gained by patients in a control group. By analyzing these results and those of previous studies, we hypothesize a new robotic protocol for acute and subacute stroke patients based on both treatment modalities (in addition and in substitution).
\end{abstract}

Clinical Trial Registration: ClinicalTrials.gov; NCT01102309, "Robot-assisted rehabilitation of the upper limb in acute and subacute poststroke patients"; http://clinicaltrials.gov/ct2/show/ NCT01102309.

Key words: cerebrovascular accident, hemiparesis, recovery of function, rehabilitation, robot, robotics, stroke, subacute therapy, training, upper limb.

\section{INTRODUCTION}

Stroke has a considerable social impact, leaving survivors with residual impaired arm function and disability in activities of daily living (ADLs). Recovery is partial in 85 percent of stroke survivors [1], about 35 percent of whom are left with a major disability; traditional rehabilitation programs leave about 30 to 60 percent still without functional use of the paretic/plegic arm [2-3]. Moreover, the number of people requiring rehabilitation treatment after stroke is rapidly growing owing to population aging [4]. The aim of rehabilitation in subjects with hemiplegia is to promote recovery of lost function, independence, and early reintegration into social and domestic life. Clinical

\footnotetext{
Abbreviations: $3-\mathrm{D}=$ three-dimensional, $\mathrm{ADL}=$ activity of daily living, $\mathrm{CG}=$ control group, $\mathrm{DOF}=$ degree of freedom, $\mathrm{EG}=$ experimental group, $\mathrm{FAT}=$ Frenchay Arm Test, $\mathrm{FM}=$ Fugl-Meyer, FM-SEC = Fugl-Meyer Shoulder, Elbow, and Coordination subsections, FM-tot $=$ Total Fugl-Meyer score, FMWH $=$ Fugl-Meyer Wrist and Hand subsections, MAS = Modified Ashworth Scale, m-FIM = motor-Functional Independence Measure, MIME = Mirror Image Movement Enabler, MRC = Medical Research Council, NeReBot $=$ Neuro-RehabilitationroBot, RCT = randomized controlled trial.

*Address all correspondence to Giulio Rosati, PhD; University of Padua, DIMEG, Via Venezia, 1 Padova I-35131, Italy; +39-049-8276809; fax: +39-049-8276816.

Email: giulio.rosati@unipd.it

DOI:10.1682/JRRD.2010.04.0063
} 
studies have reported better motor outcome with various sensorimotor approaches, including repetitive intensive mobilization [5], forced use of the paretic limb or constraint-induced movement therapy [6], biofeedback [7], functional electrical stimulation [8], and transcranial magnetic stimulation [9]. (A comparative analysis of different therapeutic approaches can be found in Langhorne et al. [10].) The available scientific literature suggests that poststroke rehabilitation intervention is significantly more effective when it is delivered in the early phase of recovery ( $<6$ months). Evidence supports that better functional outcome is determined by rehabilitation that is initiated promptly [11] and based on intensive, especially multisensory, stimulation [12-13]. This kind of stimulation is associated with increased adaptive plasticity of the brain in the early poststroke stages [14-17].

The use of robotic systems to complement standard poststroke multidisciplinary programs is a recent approach that looks very promising; robotic devices can provide high-intensity, repetitive, task-specific, interactive treatment of the impaired limb (passive and/or activeassisted exercises) and can objectively and reliably monitor patients' motor progress, measuring changes in movement kinematics and forces [18-19]. It is appropriate to consider the robot as an advanced tool to be used under the therapist's direction-a tool that can implement relatively simple repetitive and labor-intensive therapies. Clinical decisions should be managed by the rehabilitation team and, when appropriate, planned and executed on the robot; this approach should be part of an integrated set of tools that also includes simpler nonrobotic approaches [20-21].

In a recent Cochrane review, Mehrholz et al. showed that the use of electromechanical devices in rehabilitation may not significantly improve ADLs, although they did find evidence that upper-arm motor function and strength may improve [22]. Thus, the actual role of robot-assisted therapy in poststroke rehabilitation remains to be clarified, and to date, no guidelines exist on how the design and use of such devices might increase their efficacy [23]. One domain to be explored is acute-phase robotic therapy. So far, many robotic devices have been designed to deliver arm therapy in individuals with stroke [24], but only a few of them-notably, the MIT-Manus [25-26], the Mirror Image Movement Enabler (MIME) [27-28], the Bi-Manu-Track [29], and the Neuro-RehabilitationroBot (NeReBot) [30-32] — have been tested in at least one randomized controlled trial (RCT) on patients during the acute or subacute phases of their stroke. ${ }^{*}$ The results of those studies show that early robotic training of the upper limb can improve ADLs significantly more than chronic-phase training [22]. This result, corroborated by the general finding that poststroke rehabilitation is more effective when delivered in the early phase of recovery, suggests that early robotic therapy should be further investigated.

The main facts and figures of the first RCTs on early robotic stroke rehabilitation are summarized in Table 1. The MIT-Manus is a 2 degree of freedom (DOF) robot that assists shoulder and elbow movement by guiding the patient's hand in the horizontal plane [25-26]. A study in the subacute phase (starting in the fourth week after stroke on average) of 56 patients with moderate to severe poststroke hemiparesis investigated whether additional sensorimotor training enhanced motor outcome. The patients who received standard multidisciplinary poststroke rehabilitation and robotic training in addition to traditional therapy (at least 25 hours, 4-5 hours a week for 5 weeks) presented less impaired shoulder and elbow function and greater recovery of ADL functions than did patients who received no additional therapy [33].

The MIME [27-28] is a 6 DOF robotic arm developed for unilateral or bilateral shoulder and elbow treatment. In one RCT [34], 24 subacute stroke subjects were trained with the MIME (1 hour a day for 4 weeks, starting in the 11th week after stroke on average). In this study, the robot-assisted treatment (with bilateral, unilateral, and combined bilateral and unilateral modalities) was compared with conventional therapy (six patients). The results showed that the combined unilateral and bilateral robotic training had advantages over conventional therapy, producing larger improvements on a motor impairment scale and on a measure of abnormal synergies. However, gains in all treatment groups were equivalent at the 6-month follow-up.

The Bi-Manu-Track was designed specifically to train distal arm movements in bilateral passive and active mode through practice of elbow pronation and supination

\footnotetext{
*One reason for this is that early intervention is more challenging than postacute and chronic-phase treatment, because acute patients are usually severely impaired and confined to a hospital bed, may lack sufficient postural control to be treated in an upright position, and may need emergency treatment [20]. Another point is that spontaneous recovery may be a confounding variable in acute and subacute clinical trials.
} 
Table 1.

Acute- and subacute-phase randomized controlled trials on robotic therapy.

\begin{tabular}{|c|c|c|c|c|}
\hline Characteristic & Volpe et al., 2000 [1] & Hesse et al., 2005 [2] & Lum et al., 2006 [3] & Masiero et al., 2007 [4] \\
\hline Device Used & MIT-Manus & Bi-Manu-Track & MIME & NeReBot \\
\hline Time Poststroke (EG/CG) & $22 \pm 5 \mathrm{~d} / 26 \pm 1 \mathrm{~d}^{\dagger}$ & $5 \pm 1 \mathrm{wk} / 5 \pm 1 \mathrm{wk}^{\dagger}$ & 1-5 mo (11 wk average) & $<1 \mathrm{wk}$ \\
\hline Treatment Duration (wk) & 5 & 6 & 4 & 5 \\
\hline Robotic Modality (Addition/Substitution) & Addition & Substitution & Substitution & Addition \\
\hline Target of Robotic Training & Proximal & Distal & Proximal & Proximal \\
\hline $\begin{array}{l}\text { Type of Robotic Training } \\
\text { (Unilateral/Bilateral) }\end{array}$ & Unilateral & Bilateral & Mixed & Unilateral \\
\hline Type of Robotic Exercise & Planar & Fixed Point ${ }^{*}$ & $3-\mathrm{D}$ & 3-D \\
\hline CG Therapy & Placebo & FES & Conventional & Conventional + Placebo \\
\hline Sample Size (EG/CG) & $30 / 26$ & $22 / 22$ & $24 / 6$ & $17 / 18$ \\
\hline Age, yr (EG/CG) & $62 \pm 2 / 67 \pm 2^{\dagger}$ & $65 \pm 12 / 64 \pm 12^{\dagger}$ & $67 / 60$ & $63 \pm 11 / 67 \pm 12^{\dagger}$ \\
\hline Etiology (ISCH/HEM) & $49 / 7$ & $40 / 4$ & Not Stated & $35 / 0$ \\
\hline Sex, F/M (EG; CG) & $14 / 16 ; 12 / 14$ & $12 / 10 ; 12 / 10$ & $8 / 16 ; 2 / 4$ & $7 / 10 ; 7 / 11$ \\
\hline Disabled Limb, L/R (EG; CG) & $17 / 13 ; 14 / 12$ & 14/8; 11/11 & $11 / 13 ; 2 / 4$ & $4 / 11 ; 5 / 10$ \\
\hline FM at Baseline (EG/CG) & $6.0 \pm 2.5 / 5.0 \pm 1.0^{\dagger}$ & $7.9 \pm 3.4 / 7.3 \pm 3.3^{\dagger}$ & $29 / 26^{\ddagger}$ & $8.0(4.7-18.2) / 6.0(4.0-22.5)^{\S}$ \\
\hline Outcome: Motor Function & $0.68(0.14,1.22)^{\mathbb{9}}$ & $1.20(0.55,1.85)^{\mathbb{\top}}$ & $0.24(-0.66,1.14)^{9}$ & $0.52(-0.16,1.19)^{9}$ \\
\hline Outcome: ADLs ${ }^{* *}$ & $1.67(1.05,2.29)$ & $0.35(-0.25,0.95)^{9}$ & $-0.27(-1.17,0.62)^{9}$ & $0.77(0.08,1.46)^{9}$ \\
\hline
\end{tabular}

${ }^{*}$ Forearm prosupination and wrist flexion extension.

${ }^{\dagger}$ Mean \pm standard deviation.

${ }^{\ddagger}$ Estimated mean values.

$\S$ Median (upper and lower quartiles).

"Effect size (95\% confidence interval).

** All studies used Functional Independence Measure as functional outcome measure except Hesse et al. [2], which used Barthel Index Score.

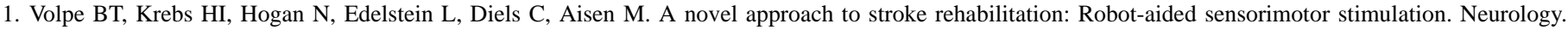
2000;54(10):1938-44. [PMID: 10822433]

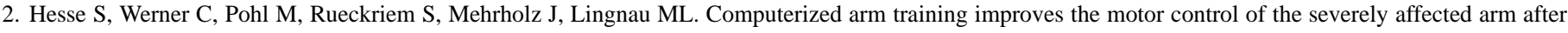
stroke: A single-blinded randomized trial in two centers. Stroke. 2005;36(9):1960-66. [PMID: 16109908] DOI:10.1161/01.STR.0000177865.37334.ce

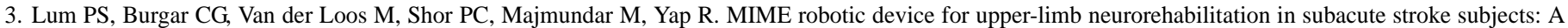
follow-up study. J Rehabil Res Dev. 2006;43(5):631-42. [PMID: 17123204] DOI:10.1682/JRRD.2005.02.0044

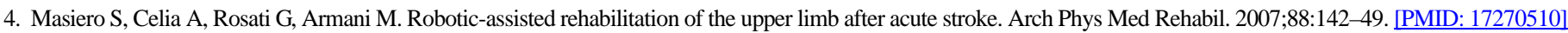
DOI:10.1016/j.apmr.2006.10.032

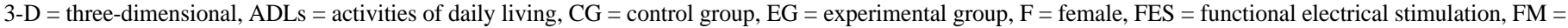

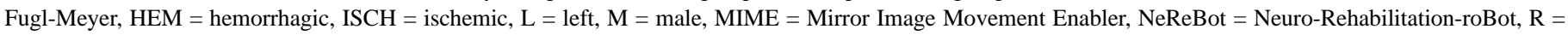
right.

as well as wrist flexion and extension in a mirror or parallel fashion [29]. A multicenter study of this device included 44 severely affected subacute stroke patients [35] with an initial average Fugl-Meyer (FM) assessment between 7 and 8 (0-66, see Table 1) and no volitional activity of the wrist and finger extensors. For 6 weeks, starting in the fifth week after stroke on average, the randomly assigned patients were trained either with the robot or with electrical stimulation of the paretic wrist extensors. Over time, both groups significantly improved their upper-limb motor control and power; the between-group comparison revealed superior results in the robot-trained group both at the end of the study and at the 3-month follow-up. Interestingly, both the proximal (0-42) and distal (0-24) FM subscores improved evenly in the robottrained group, showing that treatment effect was general- ized. The authors attributed the greater improvements in the robot-trained group to the greater number of repetitions and the bilateral approach.

At the University of Padua in Italy, we designed and developed the NeReBot, a device for treatment of poststroke upper-limb impairments in the acute and subacute phases of rehabilitation [30-32]. In the first NeReBot trial [21], the additional training delivered by NeReBot consisted of 25 daily interventions (starting within the first week poststroke) divided into 2 sessions a day for a total training time of 4 hours a week for 5 weeks, whereas the control group (CG) received similar exposure to the robot (two 30-minute sessions a week) except that the exercises were performed with the unimpaired limb. A comparison of the two groups revealed that at the end of training, the patients in the experimental group 
(EG) had greater improvements in motor and functional recovery. At the 3-month poststroke follow-up, the EG continued to show significant improvements in motor and functional recovery compared with the patients in the CG, and after 8 months poststroke, the EG sustained the gains recorded during the first follow-up.

By analyzing the data reported in Table 1, one will notice that the studies that obtained the largest effect sizes on ADL measures are the two that used robotic intervention in addition rather than in substitution of conventional upper-limb therapy [21,33]. Other common aspects of both studies are stroke severity (very small baseline FM scores), short time poststroke ( $<1$ week and 3 weeks on average), and the proximal and unilateral approach. A major difference is the type of exercises implemented: the MIT-Manus is a planar device, whereas the NeReBot has a three-dimensional (3-D) workspace.

In this new study, we tested the NeReBot in substitution of conventional (nonrobotic) proximal upper-limb exercise. Our working hypothesis was that in hemiparetic subacute stroke patients, NeReBot training could efficaciously support standard rehabilitation treatment by substituting the upper-limb portion of the conventional protocol, yielding comparatively similar results with respect to standard rehabilitation.

\section{METHODS}

\section{Robotic Device: NeReBot}

The NeReBot is a 3 DOF robotic device for upper limbs that can be easily used in the acute phase thanks to its portability and usability at bedside. The robot is based on direct-drive wire actuation: three actuated nylon cables are used to sustain and move the patient's forearm, which is fastened onto a rigid splint (Figure). Compared with the devices characterized by a rigid structure, this solution provides many benefits, mainly: lower costs, reduced complexity (3-D trajectories can be exercised despite the limited number of actuators), high robot compliance, and high reliability and safety [32]. The NeReBot can (1) help the patient perform spatial movements (flexion and extension, pronation and supination, adduction and abduction, circular) of shoulder and elbow, (2) be easily moved to the hospital room, and (3) be used to intervene on patients in not only the sitting but also the supine position. The Figure shows the NeReBot during an abduction-adduction exercise with a patient in the supine position.

The NeReBot can be programmed to perform repetitive assistive movements of the proximal upper limb (shoulder and elbow), simulating a hand-over-hand therapy with imperceptible differences in the patient's sensorimotor experience. Each exercise is recorded by manually moving the patient's forearm along a set of waypoints chosen by the therapist (learning phase). At the end of this phase, the acquired positions are interpolated to obtain a very comfortable 3-D motion of the patient's upper limb (therapy phase). During the therapy phase, the therapist instructs the patient to anticipate the trajectory, producing an active contribution to the movement of the limb. At the same time, the patient can experience voluntary movements in different directions with

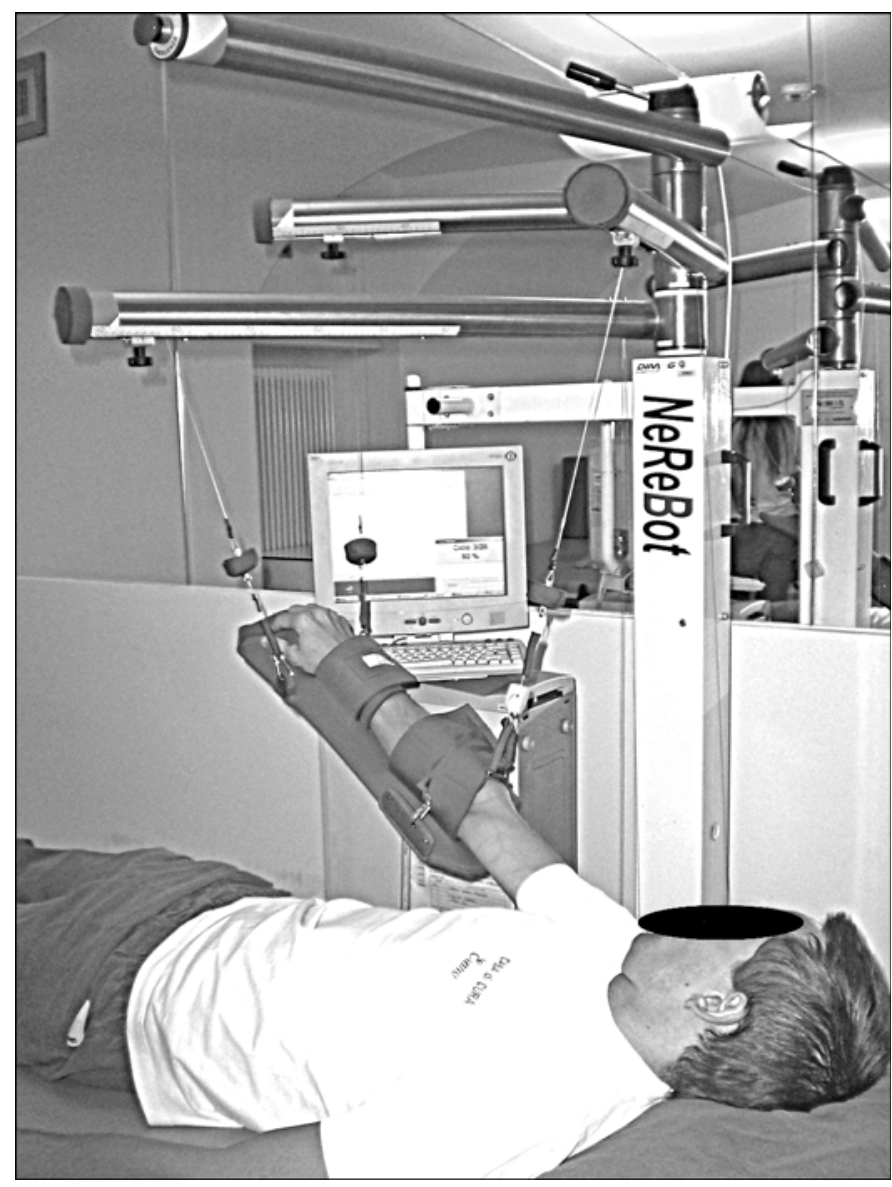

Figure.

Neuro-Rehabilitation-roBot (NeReBot) training with patient in supine position. 
respect to the preset trajectory (e.g., the horizontal direction during a shoulder abduction-adduction exercise). These movements are allowed by the robot, thanks to underactuation. In fact, only three cables are used to control the motion of a 5 DOF kinematic chain [30]. In this way, robot-patient interaction becomes highly compliant, giving the patient the very comfortable sensation of being guided through an assisted hand-over-hand therapy.

The NeReBot provides basic visual and auditory feedback to the patient. A beep signals the start and the end of each point-to-point portion of the exercise. Visual feedback consists of a static 3-D image of a virtual upper limb on which three arrows parallel to the cables show the desired direction of arm motion. Thanks to a recent upgrade, in the new trial a score proportional to the subject's effort was provided to the subject, ${ }^{*}$ both as a bar and a number displayed on the screen. In this way, we aimed to further motivate the patient to produce active motion during NeReBot training, since active movement training has far more beneficial effects on cortical reorganization and treatment outcome than passive movements.

\section{Population}

Subacute subjects after a first, single ischemic or hemorrhagic cerebrovascular accident (within 20 days) with upper-limb impairment (hemiparesis or hemiplegia) were included in the study. The following inclusion criteria were adopted: (1) diagnosis of recent single-sided stroke (ischemic or hemorrhagic) demonstrated by brain computerized axial tomography or nuclear magnetic resonance, (2) sufficient cognitive and language capacities to understand the operator's instructions (Modified MiniMental State Examination score >18) [36], (3) paralysis or paresis (Motor Power score between 8 and 12) with no ability for active movement against gravity or weak resistance [33]. ${ }^{\dagger}$ The following exclusion criteria were adopted for the study: (1) cardiovascular instability (severe, uncontrolled hypertension; severe coronary artery disease; etc.) or orthopedic or neurological conditions; (2) multiple cerebrovascular lesions (usually result of many past ischemic episodes); (3) early appearance of marked spasticity ( $\geq 3$ s Ashworth Scale) [37]; (4) upper-

\footnotetext{
*Subject's effort is estimated by subtraction of the sum of actual cable tensions (actual robot effort) from that recorded in the same point in the first repetition of the exercise, during which the subject was instructed to remain passive (maximum robot effort). The resulting value, once divided by the latter, is displayed as a percentage.
}

limb joint pain or limitations to range of motion that would have limited the subject's ability to complete the protocols; (5) severe neuropsychological impairment (global aphasia, severe attention deficit, or severe space inattention), because the patient needed to be able to follow instructions; (6) age $>85$ years or $<18$ years.

This study was approved by the Ethics Committee for clinical trials of the Provincial Agency for Health Services of Trento (Italy). Informed consent was obtained from all patients (21 subjects) and from one family member before randomized assignment to the EG $(n=11)$ or the CG $(n=10)$. Randomization was achieved with use of a sequence of computer-generated random numbers.

\section{Intervention}

The study tested a protocol that used robots as an alternative to the standard treatment for 35 percent of the total daily treatment time. Both groups received a total daily treatment time of 120 minutes for 5 days a week for 5 weeks. CG patients performed the conventional functional rehabilitation for 80 minutes a day (including proprioceptive exercises, functional reeducation, gait training, occupational therapy, and passive and active-assisted mobilization of the hand and wrist) but without specifically exercising the proximal paretic arm. The proximal paretic arm was exercised during a separate 40-minute session. For the EG, conventional treatment of the proximal paretic arm was substituted with NeReBot training for 40 minutes a day (divided into two 20-minute sessions). Robotic training was administered under the supervision of a physiotherapist, who defined the exercises (flexion and extension, adduction and abduction, pronation and supination, circular) according to the protocol, adapted cable velocities (range: $5-60 \mathrm{~mm} / \mathrm{s}$ ) to the specific exercise and the patient's progress (to keep the exercises challenging) and, in agreement with the rehabilitation team, defined the treatment schedule weekly. The same therapist supervised the NeReBot training for all EG patients, whereas a different therapist delivered standard rehabilitation to all patients.

\footnotetext{
${ }^{\dagger}$ Motor Power (maximum score $=20$ ) measures strength in the proximal muscles of the arm, specifically grading shoulder flexors and abductors and elbow flexors and extensors on the standard 6-point scale in which 0/5 means no contraction, 1/5 trace contraction, 2/5 active movement possible with gravity eliminated, 3/5 antigravity strength, 4/5 function reduced but adequate to overcome some resistance, and 5/5 normal strength.
} 
All robot treatment sessions consisted of a sequence of motor tasks followed by a short resting phase. The patients were asked to perform five to seven exercise cycles lasting nearly 3 minutes each, followed by a 30 60 -second resting period; each session lasted 20 minutes. They performed the movements slowly to avoid abnormal muscle activity that might cause pain or injury of the paretic muscles. At the start of each therapy session, the clinician examined arm impairment to investigate motor function recovery and pain or other complications; placed the patient's forearm together with the wrist and hand in a neutral position in the splint; and identified the optimal path and rest positions for the patient within robot workspace, related to the individual stage of recovery, to fully exploit the patient's residual motor skills and provide spatial stimulation. All patients sat on a chair/wheelchair ${ }^{*}$ fitted with seat belts to limit torso movements and prevent falling. Patients were instructed to actively contribute to the exercise according to the goal movements. During the robot therapy session, the therapist verbally encouraged the patient to increase effort while a score proportional to patient effort was displayed in the form of a bar and of a number on the screen. Treatment was completed in the same rehabilitation center for all the recruited subjects during hospitalization, and no patient underwent rehabilitative treatment during follow-up.

\section{Measures}

All patient assessments were performed by the same blinded clinician, who had previously attended a training course qualifying him or her to use the scales, was not directly involved in the delivery of either robot-aided or standard rehabilitation therapy within the study, and did not know which patients had been enrolled in the EG and the CG. Assessments included a number of clinical evaluations immediately before treatment start (baseline), immediately posttreatment (5 weeks after treatment start), and 3 months after the end of treatment (follow-up).

The following internationally validated motor and functional rating scales were used:

1. Medical Research Council (MRC) [38]—Used to rate the strength of the paretic arm during five actions: shoulder abduction (MRC deltoid), elbow flexion

\footnotetext{
* The use of the NeReBot at bedside is recommended in the very first days after stroke. In this study, patients started NeReBot training 10 days poststroke on average and all could be treated in the sitting position.
}

(MRC biceps), elbow extension (MRC triceps), wrist flexion (MRC wrist flexors), and extension (MRC wrist extension).

2. FM assessment [39]—Used to measure motor impairment of the upper limb; we considered the Shoulder, Elbow, and Coordination subsections (FM-SEC $=42$ / $66)$, the Wrist and Hand subsections (FM-WH $=24$ / $66)$, and the total FM score $($ FM-tot $=66 / 66)$.

3. Motor-Functional Independence Measure (m-FIM), a subsection of Functional Independence Measure [4041] — Used to measure improvements in basic motor ADLs and degree of self-care. The m-FIM may not be a good measure of upper-limb function or ability; however, we included it in the protocol to allow comparison with other studies.

4. Modified Ashworth scale (MAS) [37]—Used to test for hypertonia in several upper-limb joints. A higher score on the MAS indicates higher tone, so a lower score indicates lower, abnormal tone.

5. Frenchay Arm Test (FAT) [42]—Used to evaluate five ADLs; reflects both hand and arm functions.

6. Box and Block Test of manual dexterity [43]—Used to assess upper-limb disability.

7. Tolerability of treatment-Evaluated by noting the number of medical complications in the two groups (shoulder-hand syndrome, shoulder pains) and the degree of acceptance of the robotic training rated on a visual analog scale $(0=$ poor acceptance and $10=$ maximum acceptance).

\section{Statistical Approaches}

The baseline characteristics of patients in the CG and EG were compared by chi-square tests (nominal data) or unpaired $t$-tests for independent samples (continuous data). Before-after training improvements in each group were tested for statistical significance through Wilcoxon matched-pairs test after the end of rehabilitation therapy and at the 3-month follow-up. The Mann-Whitney $U$-test was used to identify significant differences between the two groups in average gains in scores on motor impairment (FM-SEC, FM-WH, FM-tot, MRC) and functional disability (m-FIM, FAT, Box and Block, MAS). Statistical significance was set at $p \leq 0.05$. The statistics were processed using SPSS version 17.0 (SPSS, Inc; Chicago, Illinois). 


\section{RESULTS}

Demographic characteristics and baseline motor and functional assessments of the 21 subjects enrolled are showed in Table 2. The EG $(n=11)$ and CG $(n=10)$ were comparable with respect to age, sex, side of stroke, and type of stroke at rehabilitation admission. NeReBot training of EG patients started on average 10.1 days after stroke (range $=4-15$ ). The results at the end of rehabilitation treatment and at the 3-month follow-up are summarized in Table 3. Before-after training improvements in motor and functional scales within each group were all statistically significant at the end of robot therapy. Most of the gains at follow-up were statistically significant as well, especially in the EG. In both groups, all or most muscles tested were stronger at the end of therapy and at follow-up (MRC results), motor impairment and abnormal hypertonia were reduced (FM and MAS results), and basic ADLs and dexterity were improved (m-FIM, FA-T, and Box and Block Test results). The between-group comparison revealed no significant difference between
EG and CG improvements at the end of robot therapy and at follow-up, with almost all the $p$-values considerably greater than 0.05 .

No differences were found between the two groups in terms of joint- or tendon-related pain in the shoulder, wrist, or hand or any other complications, including shoulder-hand syndrome. Only one CG patient developed a complication (shoulder-joint pain) but it did not influence performance of the rehabilitation program. The questionnaire administered to the EG patients at the end of robot therapy showed that this form of intervention was well accepted and tolerated by the patients (mean score $=8.1 / 10$ ), as already reported by the first study. All patients in the EG were in favor of including NeReBot training in the poststroke rehabilitation program.

* Only MRC wrist flexor at the end of therapy was significantly higher in the CG. However, this result did not persist at the 3-month followup. FA-T at the 3-month follow-up was also close to significance.

Table 2.

Patients’ demographic and clinical characteristics at baseline.

\begin{tabular}{|c|c|c|c|}
\hline Characteristic & $\begin{array}{c}\text { EG } \\
(n=11)\end{array}$ & $\begin{array}{c}C G \\
(n=10)\end{array}$ & $p$-Value \\
\hline$\overline{\text { Age }(\mathrm{yr})^{*}}$ & $72.4 \pm 7.1$ & $75.5 \pm 4.8$ & 0.48 \\
\hline $\operatorname{Sex}(\mathrm{M} / \mathrm{F})^{\dagger}$ & $9 / 2$ & $7 / 3$ & 1.00 \\
\hline Stroke Side $(\mathrm{R} / \mathrm{L})^{\dagger}$ & $9 / 2$ & $8 / 2$ & 1.00 \\
\hline Stroke Type (ISCH/HEM) ${ }^{\dagger}$ & $9 / 2$ & $9 / 1$ & 1.00 \\
\hline Time Between Stroke and Treatment Start (d) ${ }^{*}$ & $10.1 \pm 4.5$ & $12.5 \pm 5.2$ & 0.30 \\
\hline MRC Biceps ${ }^{\ddagger}$ & $3.0(2.2-4.0)$ & $3.0(2.0-4.0)$ & 0.78 \\
\hline MRC Triceps ${ }^{\ddagger}$ & $3.0(2.0-4.0)$ & $3.0(2.0-3.7)$ & 0.44 \\
\hline MRC Wrist Flexor ${ }^{\ddagger}$ & $3.0(0.2-4.0)$ & $3.0(0.5-3.7)$ & 0.71 \\
\hline MRC Wrist Extensor ${ }^{\ddagger}$ & $3.0(2.2-3.7)$ & $3.0(0.5-4.0)$ & 0.81 \\
\hline FM-SEC $^{\ddagger}$ & $22.0(15.2-28.5)$ & $22.5(4.5-27.5)$ & 0.59 \\
\hline Box and Block Test ${ }^{\ddagger}$ & $12.5(2.0-16.0)$ & $8.5(0-15.5)$ & 0.64 \\
\hline $\mathrm{FA}-\mathrm{T}^{\ddagger}$ & $3.0(0.2-3.7)$ & $1.5(0.0-4.7)$ & 0.90 \\
\hline MAS $\ddagger$ & $0.0(0.0-0.2)$ & $0.0(0.0-0.3)$ & 0.22 \\
\hline
\end{tabular}

*Mean \pm standard deviation.

${ }^{\dagger}$ No.

${ }^{\ddagger}$ Median (upper and lower quartiles).

CG = control group, EG = experimental group, F = female, FA-T = Frenchay Arm Test, FM = Fugl-Meyer, FM-SEC = FM Shoulder, Elbow, and Coordination subsections, FM-tot = total FM score, FM-WH = FM Wrist and Hand subsections, HEM = hemorrhagic, ISCH = ischemic, $\mathrm{L}=$ left, $\mathrm{M}=$ male, MAS $=$ Modified Ashworth Scale, m-FIM = motor-Functional Independence Measure, MRC = Medical Research Council, $\mathrm{R}$ = right. 
Table 3.

Average gains in scores (mean \pm standard deviation) at end of robot therapy and 3-month follow-up, and comparison between experimental group (EG) and control group (CG) gains (Mann-Whitney $U$-test $p$-values). Before-after training gains in each group were tested for statistical significance. Wilcoxon matched-pairs test yielded statistical significance $(p<0.05)$ for all gains except those marked with asterisk $(p<0.09)$.

\begin{tabular}{|c|c|c|c|c|c|c|}
\hline \multirow{2}{*}{ Evaluation } & \multicolumn{3}{|c|}{ End Robot Therapy } & \multicolumn{3}{|c|}{ 3-Month Follow-Up } \\
\hline & EG $(n=11)$ & CG $(n=10)$ & $p$-Value & EG $(n=11)$ & CG $(n=10)$ & $p$-Value \\
\hline$\overline{\text { MRC Deltoid }}$ & $0.58 \pm 0.90$ & $1.87 \pm 1.10$ & 0.08 & $1.00 \pm 0.80$ & $1.50 \pm 1.70^{*}$ & 0.82 \\
\hline MRC Biceps & $0.75 \pm 0.60$ & $1.12 \pm 0.60$ & 0.20 & $1.00 \pm 0.80$ & $1.00 \pm 0.80^{*}$ & 1.00 \\
\hline MRC Triceps & $0.85 \pm 0.50$ & $1.25 \pm 0.40$ & 0.10 & $1.00 \pm 0.80$ & $1.25 \pm 0.50$ & 0.64 \\
\hline MRC Wrist Flexor & $0.83 \pm 0.50$ & $1.70 \pm 1.10$ & 0.04 & $1.66 \pm 1.20^{*}$ & $1.25 \pm 1.20^{*}$ & 0.69 \\
\hline MRC Wrist Extensor & $0.83 \pm 0.70$ & $1.75 \pm 1.40$ & 0.16 & $1.50 \pm 1.30^{*}$ & $1.00 \pm 0.80^{*}$ & 0.65 \\
\hline FM-SEC & $7.33 \pm 4.70$ & $7.37 \pm 7.30$ & 0.91 & $7.16 \pm 4.40$ & $7.21 \pm 6.10^{*}$ & 0.91 \\
\hline FM-WH & $4.83 \pm 4.70$ & $6.50 \pm 3.60$ & 0.33 & $5.33 \pm 5.90$ & $7.00 \pm 1.10$ & 0.51 \\
\hline FM-tot & $12.16 \pm 8.30$ & $13.87 \pm 10.20$ & 0.76 & $12.50 \pm 8.90$ & $14.21 \pm 7.10$ & 0.59 \\
\hline m-FIM & $18.75 \pm 9.30$ & $24.52 \pm 11.40$ & 0.47 & $28.66 \pm 8.40$ & $34.50 \pm 15.40$ & 0.61 \\
\hline Box and Block Test & $12.33 \pm 7.00$ & $9.30 \pm 5.30$ & 0.24 & $17.32 \pm 16.10$ & $15.20 \pm 9.80$ & 0.83 \\
\hline FA-T & $1.83 \pm 1.40$ & $1.00 \pm 0.70$ & 0.23 & $1.83 \pm 1.40$ & $0.25 \pm 0.50^{*}$ & 0.058 \\
\hline MAS & $0.83 \pm 0.28$ & $0.50 \pm 0.70$ & 0.11 & $0.55 \pm 0.80$ & $0.75 \pm 1.20^{*}$ & 1.00 \\
\hline
\end{tabular}

FA-T = Frenchay Arm Test, FM = Fugl-Meyer, FM-SEC = FM Shoulder, Elbow, and Coordination subsections, FM-tot = total FM score, FM-WH = FM Wrist and Hand subsections, MAS = Modified Ashworth scale, m-FIM = motor Functional Independence Measure, MRC = Medical Research Council.

To allow comparison with the studies in Table 1, we calculated the following effect sizes (with 95\% confidence interval in parentheses) at the end of treatment: FM-tot $=-0.18(-1.03,0.68), \mathrm{m}$-FIM $=-0.53(-1.40$, $0.34), \mathrm{FA}-\mathrm{T}=0.71(-0.17,1.59)$ and Box and Block $=$ $0.47(-0.40,1.33)$.

\section{DISCUSSION}

Our results show that on both motor and functional scales, EG patients presented gains comparable to those of CG patients at the end of robot therapy, indicating a substantial equivalence between treatment groups. FM$\mathrm{WH}$ and m-FIM changes were noticeably more pronounced in the CG. This result may be explained by the fact that the EG had higher baseline scores in both measures. Also, as to the FM-WH, one should consider that the NeReBot exercises the proximal upper limb only. On the other hand, Box and Block and FA-T changes were more pronounced in the EG.

Another interesting point is that the improvements, which were clinically relevant, persisted at follow-up in both groups. Moreover, since gains in motor function

\footnotetext{
*Future studies will investigate whether adding a wrist/hand module
} to the robot will lead to greater gains. have not always been reported to bring significant improvements in the performance of basic self-care activities [40], these results are interesting because both groups showed gains in the functional scales. Gains in functional scales must be interpreted very carefully, because they may be the result of compensation rather than recovery. However, while reading functional scores, one should keep in mind that compensation strategies are more likely to be taught in standard rehabilitation programs [44] than in robotic training.

If we compare the results with those of our previous NeReBot study [21], the primary ADL measure (m-FIM) indicates that additional training is more efficacious than substitution with our device. The other functional scales used in this new study would draw a different result; however, they were not used in the first study. Also, we must remind readers that in the new study, the average gains in all scales did not significantly differ in the EG and CG, suggesting that poststroke patients exposed to early stimulation with NeReBot in substitution of standard proximal upper-limb mobilization achieve comparatively similar reductions in motor impairment and enhancements in the ability to perform ADLs. This result is consistent with the findings of the subacute-phase MIME study by Lum et al. [34], which compared three different robotic protocols with one conventional rehabilitation protocol, all sharing the same duration and intensity of treatment. The study showed a substantial equivalence of 
motor and functional gains in robot patients (as pooled in one group by Mehrholz et al. [22]) with respect to the CG. The same applies to ADL improvement for the acutephase trial of Bi-Manu-Track [35]. In this study, even though comparatively higher gains in motor function and motor strength were achieved in the robot group with respect to control patients, a substantially neutral effect size was obtained in terms of ADLs at the end of intervention [22]. It is worth noting that the three RCTs used different robotic systems, targeted different segments of the upper limb, and implemented different treatment modalities (unilateral, bilateral, mixed).

The potential equivalence of robotic and traditional interventions is suggested also by a recent study by Reinkensmeyer et al. [45], who used robotic treatment in substitution of unassisted movement training of the paretic upper limb in a chronic-phase setting. In this work, a computational model of motor plasticity in chronic stroke patients is presented (based on experimental evidence) that predicts an exponential-like motor recovery driven by practice, regardless of range or speed of the practiced movement, suggesting that robotic and nonrobotic techniques can result in similar improvement in movement ability after stroke. The same premise is supported by a recent multicenter RCT by Hesse et al. [44], who used robotic training of the upper limb with a mechanical arm trainer as an alternative to electrical stimulation of the paretic wrist extensors in subacute stroke patients. In this study, no statistically significant difference between groups was found in the primary outcome (FM scale); i.e., the mechanical arm trainer was not superior to the electrical stimulation on the impairment level despite a much higher repetition rate, more DOFs, and a bilateral approach. The authors hypothesized that this unexpected result may have been due to the different limb segment exercised in the two groups.

On the basis of the fact that different types of robotic training do not result in improved gains when used in substitution of nonrobotic rehabilitation techniques, one could also argue that the kind of therapy or robotics used does not matter: the fact that exercise practice occurs is the main determinant of treatment outcome. On the other hand, the hypothesis that early intensive exercise may have more effect on patient recovery than chronic-phase training is supported by a wide literature on nonrobotic rehabilitation techniques [46] and by the cited computational model by Reinkensmeyer et al. [45] that suggests that the differential effect on limb strength of a dose of movement practice will be greater earlier in recovery, because the learning curve is exponential-like. This finding is confirmed by the two acute- and subacute-phase studies that used robotic training in addition to conventional therapy or in comparison with placebo (one involving the MIT-Manus [33], the other the NeReBot [21]) and revealed that additional robotic treatment in the acute and subacute phases leads to comparatively higher improvements in ADLs [22]. Conversely, at least one study on additional robotic training in the chronic phase reported that gains in ADL measures were not greater in the robotic group with respect to controls [47].

\section{CONCLUSIONS}

In this article, we presented the results of an RCT in which robotic therapy was used in partial substitution of conventional upper-limb rehabilitation of acute stroke patients. By comparing these results with those of previous studies, we can summarize that robot-assisted rehabilitation of the upper limb in the acute and subacute phases may be successfully used (1) in substitution of conventional mobilization of the upper limb, because it can be at least as effective as conventional therapy, and (2) in addition to nonrobotic techniques. This second approach, in a limited number of trials, proved to be more effective when improved ADLs was the aim. Based on this premise, we hypothesize that an optimal robotic training protocol for acute and subacute stroke patients would be divided in two stages: initial additional robotic training (first stage) followed by substitution of part of the conventional therapy with the robotic exercise (second stage). In this way, the amount of treatment would be increased in the stage of recovery where improvements are likely to be greater. For such a protocol to be feasible without the need for increasing manpower in the rehabilitation facility, a proper robotic tool requiring only partial supervision during the execution of the exercises should be used, at least in the second stage. The time schedule of the protocol should then be adjusted to maximize the duration and intensity of the first stage of robotic treatment. With our device, for instance, almost one-to-one supervision is preferable in the first week of treatment, whereas two to three robots can be managed by a single therapist in the same treatment room by the second or third week. 
Another important point is that the robot has to be operated in the hospital ward, and possibly at bedside, to allow for very early intensification of the rehabilitation treatment. Moreover, a robot used in early functional poststroke training should, in terms of arm motion, perform exercises that promote more natural movements [48]. For example, a 3-D trajectory would be a desirable feature, because it promotes a large number of functional movements in different directions in space. Finally, a robotic system for acute-phase rehabilitation, while assisting the patient's movements, should guarantee a highly compliant interaction to facilitate voluntary motion and preserve the causal relationship between motor command and resulting arm motion, even when robotic assistance is provided. Our approach with the NeReBot can be considered very close to this philosophy, and our future research will aim to clarify whether a suitable mix of substitutive and additional robotic training in the early stage of recovery may lead to a favorable tradeoff between the needs for improved outcome and reduced treatment and personnel costs [49].

\section{ACKNOWLEDGMENTS}

\author{
Author Contributions: \\ Study concept and design: S. Masiero, M. Armani, G. Rosati. \\ Analysis and interpretation of data: S. Masiero, M. Armani, G. Rosati. \\ Statistical analysis: S. Masiero. \\ Study supervision: M. Armani, S. Masiero.
}

Financial Disclosures: The authors have declared that no competing interests exist.

Funding/Support: This material was based on work partially supported by the Autonomous Province of Trento, Italy, within the 20072008 Health Research Program (document No. 2008-S143-00178).

Additional Contributions: We express our deepest appreciation to all subjects who participated in this study. We would like to thank Gregorio Ferlini, MD, and Alice Chiasera, MD, from the Neuromotor Rehabilitation Unit, Eremo Hospital, Arco (Trento, Italy), who helped with patient enrollment.

Institutional Review: This study was approved by the Ethics Committee for Clinical Trials of the Provincial Agency for Health Services of Trento (Italy). Informed consent was obtained from all patients and one family member.

Participant Follow-Up: The authors plan to inform participants of the publication of this study.

\section{REFERENCES}

1. Truelsen T, Piechowski-Jówiak B, Bonita R, Mathers C, Bogousslavsky J, Boysen G. Stroke incidence and preva- lence in Europe: A review of available data. Eur J Neurol. 2006;13(6):581-98. [PMID:16796582]

DOI:10.1111/j.1468-1331.2006.01138.x

2. Gowland C. Recovery of motor function following stroke: Profile and predictors. Physiother Can. 1982;34(2):77-84.

DOI:10.3138/ptc.34.2.077

3. Kwakkel G, Wagenaar RC, Kollen BJ, Lankhorst GJ. Predicting disability in stroke-A critical review of the literature. Age Ageing. 1996;25(6):479-89. [PMID: 9003886] DOI:10.1093/ageing/25.6.479

4. World Health Organization. World Health Statistics 2008. Geneva (Switzerland): WHO Press; 2008.

5. Bütefisch C, Hummelsheim H, Denzler P, Mauritz H. Repetitive training of isolated movements improves the outcome of motor rehabilitation of the centrally paretic hand. J Neurol Sci. 1995;130(1):59-68. [PMID: 7650532] DOI:10.1016/0022-510X(95)00003-K

6. Sirtori V, Corbetta D, Moja L, Gatti R. Constraint-induced movement therapy for upper extremities in stroke patients. Cochrane Database Syst Rev. 2009;(4):CD004433. [PMID: 19821326]

7. Wolf SL, Binder-MacLeod SA. Electromyographic biofeedback applications to the hemiplegic patient. Changes in upper extremity neuromuscular and functional status. Phys Ther. 1983;63(9):1393-1403. [PMID: 6611660]

8. Popovi DB, Sinkaer T, Popovi MB. Electrical stimulation as a means for achieving recovery of function in stroke patients. NeuroRehabilitation. 2009;25(1):45-58. [PMID: 19713618]

9. Machado S, Bittencourt J, Minc D, Portella CE, Velasques B, Cunha M, Budde H, Basile LF, Chadi G, Cagy M, Piedade R, Riberio P. Therapeutic applications of repetitive transcranial magnetic stimulation in clinical neurorehabilitation. Funct Neurol. 2008;23(3):113-22. [PMID: 19152730]

10. Langhorne P, Coupar F, Pollock A. Motor recovery after stroke: A systematic review. Lancet Neurol. 2009;8(8):741-54. [PMID: 19608100] DOI:10.1016/S1474-4422(09)70150-4

11. Paolucci S, Antonucci G, Grasso MG, Morelli D, Troisi E, Coiro P, Bragoni M. Early versus delayed inpatient stroke rehabilitation: A matched comparison conducted in Italy. Arch Phys Med Rehabil. 2000;81(6):695-700. [PMID: 10857508]

12. Oujamaa L, Relave I, Froger J, Mottet D, Pelissier JY. [Rehabilitation of arm function after stroke. Literature review.] Ann Phys Rehabil Med. 2009;52(3):269-93. English, French. [PMID: 19398398]

13. The Italian Guidelines for Stroke Prevention and Treatment (SPREAD). Milan (Italy): Hypephar Group; 2007.

14. Dobkin BH. Strategies for stroke rehabilitation. Lancet Neurol. 2004;3(9):528-36. [PMID: 15324721] DOI:10.1016/S1474-4422(04)00851-8 
15. Biernaskie J, Chernenko G, Corbett D. Efficacy of rehabilitative experience declines with time after focal ischemic brain injury. J Neurosci. 2004;24(5):1245-54. [PMID: 14762143] DOI:10.1523/JNEUROSCI.3834-03.2004

16. Marsh EB, Hillis AE. Recovery from aphasia following brain injury: The role of reorganization. Prog Brain Res. 2006;157: 143-56. [PMID: 17046670] DOI:10.1016/S0079-6123(06)57009-8

17. Green JB. Brain reorganization after stroke. Top Stroke Rehabil. 2003;10(3):1-20. [PMID: 14681816] DOI:10.1310/H65X-23HW-QL1G-KTNQ

18. Prange GB, Jannink MJ, Groothuis-Oudshoorn CG, Hermens HJ, IJzerman MJ. Systematic review of the effect of robot-aided therapy on recovery of the hemiparetic arm after stroke. J Rehabil Res Dev. 2006;43(2):171-84. [PMID: 16847784] DOI:10.1682/JRRD.2005.04.0076

19. Kwakkel G, Kollen BJ, Krebs HI. Effects of robot-assisted therapy on upper limb recovery after stroke: A systematic review. Neurorehabil Neural Repair. 2008;22(2):111-21. [PMID: 17876068$]$ DOI:10.1177/1545968307305457

20. Harwin WS, Patton JL, Edgerton VR. Challenges and opportunities for robot-mediated neurorehabilitation. Proceedings of the IEEE. 2006;94:1717-26.

DOI:10.1109/JPROC.2006.880671

21. Masiero S, Celia A, Rosati G, Armani M. Robotic-assisted rehabilitation of the upper limb after acute stroke. Arch Phys Med Rehabil. 2007;88(2):142-49. [PMID: 17270510] DOI:10.1016/j.apmr.2006.10.032

22. Mehrholz J, Platz T, Kugler J, Pohl M. Electromechanical and robot-assisted arm training for improving arm function and activities of daily living after stroke. Cochrane Database Syst Rev. 2008;(4):CD006876. [PMID: 18843735]

23. Reinkensmeyer DJ, Jose AG, Marchal L, Wolbrecht ET, Bobrow JE. Some key problems for robot-assisted movement therapy research: A perspective from the University of California at Irvine. Proceedings of the IEEE 10th International Conference on Rehabilitation Robotics, 2007; 2007 Jun 13-15; Noordwijk, the Netherlands. Los Alamitos (CA): IEEE; 2007. p. 1009-15.

24. Timmermans AA, Seelen HA, Willmann RD, Kingma H. Technology-assisted training of arm-hand skills in stroke: Concepts on reacquisition of motor control and therapist guidelines for rehabilitation technology design. J Neuroeng Rehabil. 2009;6:1. [PMID: 19154570]

DOI:10.1186/1743-0003-6-1

25. Krebs HI, Hogan N, Aisen ML, Volpe BT. Robot-aided neurorehabilitation. IEEE Trans Rehabil Eng. 1998;6(1): 75-87. [PMID: 9535526] DOI:10.1109/86.662623
26. Krebs HI, Volpe BT, Aisen ML, Hogan N. Increasing productivity and quality of care: Robot-aided neuro-rehabilitation. J Rehabil Res Dev. 2000;37(6):639-52. [PMID: 11321000]

27. Burgar CG, Lum PS, Shor PC, Machiel Van der Loos HF. Development of robots for rehabilitation therapy: The Palo Alto VA/Stanford experience. J Rehabil Res Dev. 2000;37(6): 663-73. [PMID: 11321002]

28. Lum PS, Burgar CG, Shor PC. Evidence for improved muscle activation patterns after retraining of reaching movements with the MIME robotic system in subjects with poststroke hemiparesis. IEEE Trans Neural Syst Rehabil Eng. 2004;12(2):186-94. [PMID: 15218933]

DOI:10.1109/TNSRE.2004.827225

29. Hesse S, Schulte-Tigges G, Konrad M, Bardeleben A, Werner C. Robot-assisted arm trainer for the passive and active practice of bilateral forearm and wrist movements in hemiparetic subjects. Arch Phys Med Rehabil. 2003;84(6): 915-20. [PMID: 12808550] DOI:10.1016/S0003-9993(02)04954-7

30. Rosati G, Gallina P, Masiero S. Design, implementation and clinical tests of a wire-based robot for neurorehabilitation. IEEE Trans Neural System Rehabil Eng. 2007;15(4): 560-69. [PMID: 18198714] DOI:10.1109/TNSRE.2007.908560

31. Masiero S, Celia A, Armani M, Rosati G, Tavolato B, Ferraro $\mathrm{C}$, Ortolani $\mathrm{M}$. Robot-aided intensive training in poststroke recovery. Aging Clin Exp Res. 2006;18(3):261-65. [PMID: 16804374]

32. Rosati G, Gallina P, Rossi A, Masiero S. Wire-based robots for upper-limb rehabilitation. Int J Assist Robotics Mechatronics. 2006;7(2):3-10.

33. Volpe BT, Krebs HI, Hogan N, Edelstein L, Diels C, Aisen M. A novel approach to stroke rehabilitation: Robot-aided sensorimotor stimulation. Neurology. 2000;54(10):1938-44. [PMID: 10822433]

34. Lum PS, Burgar CG, Van der Loos M, Shor PC, Majmundar M, Yap R. MIME robotic device for upper-limb neurorehabilitation in subacute stroke subjects: A follow-up study. J Rehabil Res Dev. 2006;43(5):631-42. [PMID: 17123204] DOI:10.1682/JRRD.2005.02.0044

35. Hesse S, Werner C, Pohl M, Rueckriem S, Mehrholz J, Lingnau ML. Computerized arm training improves the motor control of the severely affected arm after stroke: A single-blinded randomized trial in two centers. Stroke. 2005; 36(9):1960-66. [PMID: 16109908] DOI:10.1161/01.STR.0000177865.37334.ce

36. Galasko D, Klauber MR, Hofstetter CR, Salmon DP, Lasker B, Thal LJ. The Mini-Mental State Examination in the early diagnosis of Alzheimer's disease. Arch Neurol. 1990;47(1):47-52. [PMID: 2294894$]$ 
37. Bohannon RW, Smith MB. Interrater reliability of a modified Ashworth scale of muscle spasticity. Phys Ther. 1987; 67(2):206-7. [PMID: 3809245]

38. Medical Research Council. Aids to the examination of the peripheral nervous system. London (UK): HMSO; 1976.

39. Fugl-Meyer AR, Jääskö L, Leyman L, Olsson S, Steglind S. The post-stroke hemiplegic patient. 1. A method for evaluation of physical performance. Scand J Rehabil Med. 1975; 7(1):13-31. [PMID: 1135616]

40. Tesio L, Granger CV, Perucca L, Franchignoni FP, Battaglia MA, Russell CF. The FIM instrument in the United States and Italy: A comparative study. Am J Phys Med Rehabil. 2002;81(3):168-76. [PMID: 11989512] DOI:10.1097/00002060-200203000-00003

41. Functional Independence Measure: versione Italiana. Manuale d'uso. Ricerca Riabil. 1992;2(suppl):1-44.

42. Wade DT. Measurement in neurological rehabilitation. Oxford (UK): Oxford University Press; 1992.

43. Desrosiers J, Bravo G, Hébert R, Dutil E, Mercier L. Validation of the Box and Block Test as a measure of dexterity of elderly people: Reliability, validity, and norms studies. Arch Phys Med Rehabil. 1994;75(7):751-55. [PMID: 8024419]

44. Hesse S, Werner C, Pohl M, Mehrholz J, Puzich U, Krebs HI. Mechanical arm trainer for the treatment of the severely affected arm after a stroke: A single-blinded randomized trial in two centers. Am J Phys Med Rehabil. 2008;87(10): 779-88. [PMID: 18806506] DOI:10.1097/PHM.0b013e318186b4bc

45. Reinkensmeyer DJ, Maier MA, Guigon E, Chan V, Akoner OM, Wolbrecht ET, Cramer SC, Bobrow JE. Do robotic and non-robotic arm movement training drive motor recovery after stroke by a common neural mechanism? Experimental evidence and a computational model. Conf Proc IEEE Eng Med Biol Soc. 2009;2009:2439-41. [PMID: 19965205]
46. Van Peppen RP, Kwakkel G, Wood-Dauphinee S, Hendriks HJ, Van der Wees PJ, Dekker J. The impact of physical therapy on functional outcomes after stroke: What's the evidence? Clin Rehabil. 2004;18(8):833-62.

[PMID: 15609840]

DOI:10.1191/0269215504cr843oa

47. Fazekas G, Horvath M, Troznai T, Toth A. Robot-mediated upper limb physiotherapy for patients with spastic hemiparesis: A preliminary study. J Rehabil Med. 2007;39(7): 580-82. [PMID: 17724559] DOI:10.2340/16501977-0087

48. Rosati G, Bobrow JE, Reinkensmeyer DJ. Compliant control of post-stroke rehabilitation robots: Using movementspecific models to improve controller performance. Proceedings of the ASME International Mechanical Engineering Congress and Exposition (IMECE2008); 2008 Oct 31Nov 6; Boston, MA.

49. Rosati G. The place of robotics in post-stroke rehabilitation. Exp Rev Med Devices. 2010;7(6):753-58.

[PMID: 21050086]

DOI:10.1586/erd.10.49

Submitted for publication May 3, 2010. Accepted in revised form January 24, 2011.

This article and any supplementary material should be cited as follows:

Masiero S, Armani M, Rosati G. Upper-limb robotassisted therapy in rehabilitation of acute stroke patients: Focused review and results of new randomized controlled trial. J Rehabil Res Dev. 2011;48(4):355-66.

DOI:10.1682/JRRD.2010.04.0063

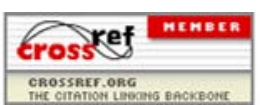

